Sophie Lalanne, Une éducation grecque. Rites de passage et construction des genres dans le roman grec ancien

Paris, Éditions la découverte, 2006, 312 pages.

Claudine Leduc

C OpenEdition

Journals

Édition électronique

URL : https://journals.openedition.org/clio/9042

DOI : $10.4000 /$ clio.9042

ISSN : $1777-5299$

Éditeur

Belin

Édition imprimée

Date de publication : 15 décembre 2008

Pagination : 275-307

ISSN : 1252-7017

Référence électronique

Claudine Leduc, « Sophie Lalanne, Une éducation grecque. Rites de passage et construction des genres dans le roman grec ancien ", Clio. Histoire, femmes et sociétés [En ligne], 28 | 2008, mis en ligne le 16 décembre 2008, consulté le 22 avril 2022. URL : http://journals.openedition.org/clio/9042 ; DOI : https://doi.org/10.4000/clio.9042

Ce document a été généré automatiquement le 22 avril 2022

Tous droits réservés 


\section{Sophie Lalanne, Une éducation grecque. Rites de passage et construction des genres dans le roman grec ancien}

Paris, Éditions la découverte, 2006, 312 pages.

Claudine Leduc

1 Dans cet ouvrage, qui fut à l'origine une thèse universitaire, "Tout a été fait pour faciliter la lecture par les non-hellénistes » déclare l'auteure dans l'introduction (p. 17). Pari tenu ! Cette étude, très savante, très structurée, écrite dans une langue limpide et élégante est d'une lecture si facile et si plaisante qu'elle vous donne la sensation fort agréable de partager son intelligence.

2 Le corpus très homogène de Sophie Lalanne est composé de cinq romans, Callirhoé de Chariton d'Aphrodisias, les Ephésiaques de Xénophon d'Ephèse, Daphnis et Chloé ou les Pastorales de Longus, Leucippé et Clitophon d'Achille Tatius, les Ethiopiques d'Héliodore. Ce sont les cinq survivants d'un genre littéraire - le roman grec - dont la période de floraison, relativement restreinte «s'étendrait du milieu du Ier siècle ap. J.-C. aux alentours de 230 ap. J.-C., avec une concentration notable au II siècle. Ce phénomène recouvrirait pour l'essentiel celui de la Seconde Sophistique selon Philostrate» (p. 57). Ce mouvement, dont sont tributaires tous les auteurs de l'époque, est au cœur de «la renaissance " de la culture grecque à l'époque des Antonins et des Sévères, une renaissance qui s'appuie «sur la redécouverte des auteurs anciens pour promouvoir de nouvelles formes de création et de pensée » (p. 41).

Tous les romans grecs racontent la même histoire d'amour et d'aventures, avec des variantes qui, bien que nombreuses, ne modifient pas la structure d'ensemble : un jeune homme et une jeune fille, issus de la plus haute aristocratie de leur cité, tombent victimes d'un coup de foudre partagé ; ils s'avouent leur amour et, dans certains cas, sont mariés par leurs parents, mais sont aussitôt séparés par un accident, un naufrage ou un enlèvement qui s'accompagne toujours d'un voyage maritime ; ce départ donne le signal d'une longue série d'aventures trépidantes qui 
les mènent aux confins de la terre habitée ; au terme de cette mise à l'épreuve, les deux adolescents sont réunis et rentrent triomphalement dans la cité de leurs pères (p. 47).

3 Sophie Lalanne démontre de façon plus que convaincante que ces fictions, qui s'affranchissent apparemment de toute contrainte temporelle (l'histoire de Callirhoé est censée se dérouler au IV ${ }^{\mathrm{e}} \mathrm{s}$ av. J.-C) et spatiale (il n'est jamais question de Rome et toute la terre habitée sert de cadre aux épreuves des amoureux), «constituent une source historique encore largement sous-exploitée » (p. 45).

4 S. Lalanne étudie d'abord le contexte historique dans lequel a été élaboré et diffusé ce nouveau genre littéraire. «Il s'étendit probablement à toute la partie hellénophone de l'empire » romain et en particulier dans ses provinces orientales (p. 58). Son monde est celui des « cités grecques » d'Asie mineure qui, grâce à la pax romana, ont retrouvé leur prospérité d'antan et sont en contact avec toutes les provinces de l'empire. «Tout en évoluant vers un régime politique de plus en plus oligarchique et fermé, ce qu'on appelle un régime de notables, elles surent conserver l'indépendance de leurs institutions et de leurs décisions concernant la vie de la communauté civique "... C'est ainsi que l'éphébie reste la porte d'entrée dans la citoyenneté, mais son entrée étant fondée sur des critères financiers, elle est réservée à une élite (p. 29). Le roman grec est inhérent à cette « société de notables, de gens d'esprit et de lettres » qui « portent très haut les couleurs de l'hellénisme » (p. 59).

Les cinq romans survivants sont " une histoire romancée de la jeunesse », ou pour le dire avec plus de précision, des «romans de formation» de couples conjugaux appartenant à la notabilité. Héros et héroïnes sont tous jeunes, sur le point d'atteindre l'âge du mariage. Le vocabulaire est précis sur les classes d'âge. Les garçons sont pais/ enfant, meirakion/adolescent, néaniskos/tout jeune homme, néanias/néos/jeune homme, anêr/homme. Les filles sont korè/jeune fille, parthénos/vierge, gunè/femme mariée. Héros et héroïnes sont tous d'excellentes familles, y compris Daphnis et Chloé. Ces pastoureaux élevés par des esclaves se trouvent tous deux à la fin de leur aventure nantis de parents riches et bien nés.

Le caractère initiatique que revêt la formation des jeunes de la notabilité dans le roman grec fait l'objet du chapitre IV : «Éducation et rites de passage ». Le franchissement de la frontière entre l'enfance et l'âge adulte se déroule en trois phases, les trois phases mises en évidence par A. van Gennep : le temps de la séparation, et particulièrement la séparation d'avec le père, se matérialise par un changement de lieu « le plus souvent la traversée de la Méditerranée »; le temps de l'isolement est celui des épreuves subies en exil, aux marges de l'espace civilisé; le temps de l'agrégation est celui du retour triomphal dans la famille et dans la cité. Cette mise en scène du passage établit, comme l'a montré $\mathrm{P}$. Bourdieu, une ligne de partage entre ceux qui ont accès à la formation initiatique et ceux qui en sont exclus : les héros, de retour dans le monde qui est le leur, trouvent immédiatement une position élevée. L'originalité du roman grec, c'est qu'il raconte non pas le passage à l'âge adulte d'un garçon comme le font les récits d'« enfances » qu'il a hérités de la tradition poétique, mais celui d'un couple conjugal rigoureusement homogamique. Il semble bien, écrit Sophie Lalanne, que « ce soit dans cette représentation de la jeunesse que le roman grec se rapproche des realia » (p. 96). La fabrication du couple conjugal n'exprimerait-elle pas dans la fiction la façon dont les notables des cités grecques ont organisé la transmission de leurs avoirs et de leurs 
pouvoirs et construit la pérennité de leur domination sociale et politique? Quelle bonne question proposée aux spécialistes de la parenté !

7 En passant du statut d'enfant à celui d'adulte, héros et héroïnes subissent une transformation de leur personnalité. Leur courte période de probation (18 mois dans Daphnis et Chloé) est celle de la fabrication, fortement différenciée selon le sexe, d'un notable de genre masculin et d'une notable de genre féminin destinés à être unis par le mariage et à se reproduire. Sophie Lalanne étudie donc, en examinant le cas de tous les personnages, la façon dont, dans les romans de formation, on devient un homme ou une femme de haut rang. Et c'est un vrai bonheur de la lire! Le héros est souvent au départ dépourvu des qualités attendues d'un héros tel que l'exige la conception traditionnelle de la masculinité en pays grec : il est orgueilleux, pleutre, timide, pas très futé. Mais les épreuves auxquelles il est soumis sont des épreuves d'action qui vont le valoriser. Il doit partir à la recherche de l'héroïne, rivaliser avec d'autres hommes par la parole ou par les armes, se former à la sexualité et acquérir une position élevée au cours de ses pérégrinations afin que son retour dans la cité puisse lui assurer une position sociale analogue à celle de son père ou de son beau-père. L'héroïne, par sa naissance, sa beauté et sa vivacité d'esprit, occupe souvent au départ une position de premier plan. Sa mise à l'épreuve consiste à préserver sa chasteté, ainsi que l'intégrité de l'amour qu'elle porte au héros, et elle le fait, face à des hommes qui veulent l'épouser ou la violer, avec un courage que la tradition attribue aux hommes. Mais ses prouesses ne valorisent pas sa position, «les romanciers opérant un rééquilibrage des rôles sexués plus conformes aux normes en vigueur dans la société grecque » (p. 130).

8 L'analyse que fait Sophie Lalanne de ce rééquilibrage des genres est particulièrement subtile. Les personnages féminins occupent une place si importante dans ces cinq romans que certains spécialistes ont vu dans ce nouveau genre littéraire «le réceptacle d'une forme d'émancipation féminine " (p. 129). L'héroïne, au cours de la phase de passage, ne fait-elle pas preuve d'autonomie? Mais lorsqu'elle se donne en mariage et signe contrat, elle est dans la zone du passage : elle n'est plus sous la domination de son père et pas encore sous celle de son mari. Sophie Lalanne montre que le roman "renforce les attentes masculines vis-à-vis du rôle des femmes dans la société " (p. 253). Le rôle d'une femme adulte est celui de l'épouse chaste et l'arétè d'une épouse associe chasteté et soumission. La mise à l'épreuve des jeunes filles, qui «consiste principalement en un examen de leur capacité à faire des épouses fidèles » (p. 228), est peut-être une façon de gommer leur personnalité, de leur dénier identité et singularité. Pour que soit assurée dans le couple la domination du héros, il faut que sa figure s'affirme progressivement tandis que s'estompe celle de l'héroïne. Les deux mouvements sont complémentaires. le cas des deux pastoureaux en est certainement le meilleur exemple: "l'effacement progressif de Chloé a pour symétrique l'octroi à Daphnis d'une supériorité incontestable » (p. 140) qui lui assurera la domination dans sa maison et dans la cité.

9 «L'apprentissage de l'arétè par les héroïnes ne se fait pas sans une grande violence " (p. 255). La violence contre les femmes, et notamment la violence sexuelle, est une constante des textes grecs, mais elle est débordante dans le roman. Les épreuves auxquelles elles sont soumises s'accompagnent de sévices corporels décrits avec force détails, notamment dans Leucippé et Cléophon. Il ne s'agit pas seulement de voyeurisme, de perversité érotique ou de sadisme. La question doit être abordée « du point de vue des interactions sociales entre hommes et femmes» (p.258). La 
disproportion entre la violence qui frappe les héroïnes et celle qui frappe les héros " donne une information quant au niveau de coercition nécessaire pour distribuer aux uns et aux autres leur rôle dans la société » (p. 277). Si la fabrication d'un homme est le produit d'une conception contraignante de la masculinité, la fabrication d'une femme qui consiste à lui faire accepter la domination du féminin l'est bien davantage. Conclusion sous forme de réponse à la question posée par l'auteure : le roman grec est bien une source historique de première importance. 